\title{
Association of Cerebrospinal Fluid (CSF) Insulin with Cognitive Performance and CSF Biomarkers of Alzheimer's Disease
}

Stefan L.C. Geijselaers ${ }^{\mathrm{a}, \mathrm{b}}$, Pauline Aalten ${ }^{\mathrm{c}}$, Inez H.G.B. Ramakers ${ }^{\mathrm{c}}$, Peter Paul De Deyn ${ }^{\mathrm{d}}$, Annemieke C. Heijboer ${ }^{\mathrm{e}}$, Huiberdina L. Koek ${ }^{\mathrm{a}}$, Marcel G.M. OldeRikkert ${ }^{\mathrm{f}}$, Janne M. Papma ${ }^{\mathrm{g}}$, Fransje E. Reesink ${ }^{\mathrm{d}}$, Lieke L. Smits ${ }^{\mathrm{h}}$, Coen D.A. Stehouwer ${ }^{\mathrm{b}}$, Charlotte E. Teunissen ${ }^{\mathrm{i}}$, Frans R.J. Verhey ${ }^{c}$, Wiesje M. van der Flier ${ }^{\mathrm{h}}$ and Geert Jan Biessels ${ }^{\mathrm{b}, *}$ on behalf of the Parelsnoer Institute Neurodegenerative Diseases study group

${ }^{a}$ Departments of Neurology and Geriatrics Brain Centre Rudolf Magnus, University Medical Centre Utrecht, Utrecht, the Netherlands

${ }^{\mathrm{b}}$ Department of Internal Medicine and Cardiovascular Research Institute, Maastricht University Medical Centre +, Maastricht, the Netherlands

${ }^{\mathrm{c}}$ Alzheimer Centre Limburg, School for Mental Health and Neuroscience (MHeNS), Maastricht University Medical Centre +, Maastricht, the Netherlands

${ }^{\mathrm{d}}$ Department of Neurology and Alzheimer Research Centre, University of Groningen, University Medical Centre Groningen, Groningen, the Netherlands

${ }^{\mathrm{e}}$ Department of Clinical Chemistry, Endocrine Laboratory, VU University Medical Centre, Amsterdam, the Netherlands

${ }^{\mathrm{f}}$ Radboudumc Alzheimer Centre, Donders Institute for Brain, Cognition and Behaviour, Radboud University Medical Centre, Nijmegen, the Netherlands

${ }^{\mathrm{g}}$ Departments of Neurology and Radiology, Erasmus University Medical Centre, Rotterdam, the Netherlands ${ }^{\mathrm{h}}$ Alzheimer Centre Amsterdam, VU University Medical Centre, Amsterdam, the Netherlands ${ }^{\mathrm{i}}$ Department of Clinical Chemistry, Neurochemistry Laboratory and Biobank, VU University Medical Centre, Amsterdam, the Netherlands

Handling Associate Editor: Adrian Ivanoiu

Accepted 10 September 2017

\begin{abstract}
.
Background: Abnormal insulin signaling in the brain has been linked to Alzheimer's disease (AD).

Objective: To evaluate whether cerebrospinal fluid (CSF) insulin levels are associated with cognitive performance and CSF amyloid- $\beta$ and Tau. Additionally, we explore whether any such association differs by sex or APOE $\varepsilon 4$ genotype.

Methods: From 258 individuals participating in the Parelsnoer Institute Neurodegenerative Diseases, a nationwide multicenter memory clinic population, we selected 138 individuals (mean age $66 \pm 9$ years, $65.2 \%$ male) diagnosed with subjective cognitive impairment $(n=45)$, amnestic mild cognitive impairment $(n=44)$, or $\mathrm{AD}(n=49)$, who completed a neuropsychological assessment, including tests of global cognition and memory performance, and who underwent lumbar puncture. We measured CSF levels of insulin, amyloid- $\beta_{1-42}$, total (t-)Tau, and phosphorylated (p-)Tau.
\end{abstract}

\footnotetext{
*Correspondence to: Professor Geert Jan Biessels, Department of Neurology, G03.232, University Medical Centre Utrecht, PO
} 
Results: CSF insulin levels did not differ between the diagnostic groups $(p=0.136)$. Across the whole study population, CSF insulin was unrelated to cognitive performance and CSF biomarkers of AD, after adjustment for age, sex, body mass index, diabetes status, and clinic site (all $p \geq 0.131$ ). Importantly, however, we observed effect modification by sex and APOE $\varepsilon 4$ genotype. Specifically, among women, higher insulin levels in the CSF were associated with worse global cognition (standardized regression coefficient $-0.483 ; p=0.008)$ and higher $\mathrm{p}$-Tau levels $(0.353 ; p=0.040)$. Among non-carriers of the APOE $\varepsilon 4$ allele, higher CSF insulin was associated with higher t-Tau $(0.287 ; p=0.008)$ and $\mathrm{p}$-Tau $(0.246 ; p=0.029)$.

Conclusion: Our findings provide further evidence for a relationship between brain insulin signaling and AD pathology. It also highlights the need to consider sex and $\mathrm{APOE} \varepsilon 4$ genotype when assessing the role of insulin.

Keywords: Alzheimer's disease, cerebrospinal fluid, cognition, epidemiology, insulin

\section{INTRODUCTION}

Over the last decades, a growing body of evidence from in vitro experiments, animal models, and observations in patients has linked abnormal insulin signaling to Alzheimer's disease (AD) [1-4]. Multiple pathways may account for this, which have been extensively reviewed elsewhere [1-4]. Insulin is, for example, believed to interfere with the metabolism of amyloid- $\beta(\mathrm{A} \beta)$ and tau [3].

At present, brain insulin signaling cannot be measured directly in humans in vivo. Evidence of deficient insulin signaling in patients with AD therefore stems from postmortem brain studies showing fewer insulin receptors and decreased downstream signaling activity in the brains of individuals with $\mathrm{AD}$ as compared to age-matched controls [5]. To overcome the lack of direct measures, previous population-based studies used blood-derived markers of peripheral insulin resistance as a possible indicator of deficient brain signaling. These studies generally showed that hyperinsulinemia [6-8] and insulin resistance $[6,9,10]$ are associated with cognitive impairment [8-10] and risk of $\mathrm{AD}[6-8,10]$, although these associations might be limited to, or most prominent in, women [9] and non-carriers of the apolipoprotein E $\varepsilon 4$ (APOE $\varepsilon 4$ ) allele [7, 9, 10]. More recently, blood-derived markers of insulin resistance have also been related to cerebrospinal fluid (CSF) biomarkers of $\mathrm{AD}$ in cognitively asymptomatic individuals, but these associations depended on APOE $\varepsilon 4$ genotype [11, 12].

Evidently, peripheral insulin levels and insulin sensitivity may not accurately reflect the cerebral situation [13]. Transport of insulin across the blood-brain barrier decreases with increasing insulin resistance [14] and there appears to be local production of insulin in the brain [15]. This may explain why there is no clear correlation between plasma and CSF insulin levels [15]. Published data on CSF insulin in relation to $\mathrm{AD}$ are, however, scarce, show variable results, and generally (with one exception [16]) did not account for the potential modifying effects of sex and APOE $\varepsilon 4$ genotype [17-21]. At the same time, an increasing number of clinical trials have shown that intranasal insulin administration enhances memory function (in AD) (e.g., [22-26]), particularly in women and those without APOE $\varepsilon 4$ [24-26]. Here, we made use of a standardized multicenter memory clinic population to assess the associations of CSF insulin with cognitive performance and CSF biomarkers of $\mathrm{AD}$ [i.e., $A \beta_{1-42}$, Tau, and phosphorylated Tau (p-Tau)] and explored whether any such association differed by sex or APOE $\varepsilon 4$ genotype. We hypothesized that higher CSF insulin levels, likely reflecting cerebral insulin resistance, would be associated with $\mathrm{AD}$ pathology.

\section{METHODS}

\section{Study population}

This study was based on data from the Parelsnoer Institute: Neurodegenerative Diseases, a collaboration between the eight Dutch University Medical Centres (http://www.parelsnoer.org) [27]. In short, the Pearl Neurodegenerative Diseases focuses on the role of biomarkers in the early diagnosis, differential diagnosis, and prognosis of neurodegenerative diseases, in particular AD [27]. Eligible for inclusion are individuals referred to one of the eight academic memory clinics for the evaluation of cognitive problems, with a Clinical Dementia Rating scale of 0, 0.5, or 1, and a Mini-Mental State Examination (MMSE) of 20 or higher. Harmonized protocols are used to collect clinical data and biomaterials from patients who provide written informed consent [27]. Individuals are classified as having subjective cognitive impairment (SCI), mild cognitive impairment (MCI), or dementia, using a diagnostic procedure that is harmonized across the participating centers [27]. 
For the present cross-sectional analyses, we used data from the first 258 patients who were enrolled in the database between March 2009 and December 2013 and successfully underwent lumbar puncture, which is optional for participants in this study. From these, we selected those patients diagnosed with $\mathrm{AD}$ or its potential prodromal stages (i.e., amnestic MCI or SCI), thereby excluding individuals with nonamnestic MCI $(n=26)$ or other types of dementia $(n=37)$. To distinguish amnestic from non-amnestic MCI, objective memory deficits were defined as age-, sex-, and educational level-adjusted immediate or delayed recall scores on the 15 Word-Auditory Verbal Learning Test (AVLT) $[28,29]$ that were at least 1.5 standard deviation below the normative mean [30]. Individuals in whom the presence of objective deficits could not be verified due to missing data on the AVLT or educational level were excluded $(n=3)$.

CSF insulin could be determined for 160 out of the 192 patients $(83.3 \%)$ who underwent lumbar puncture and were eligible for the present study. In the other 32 patients, there was too little CSF left after $\mathrm{A} \beta_{1-42}$, Tau, and p-Tau assays. Of the 160 patients, $5(3 \%)$ had no data on APOE $\varepsilon 4$ genotype and were therefore excluded from the study sample. An additional 17 individuals were excluded because of missing data on demographic and clinical variables, primarily body mass index $(n=13)$ and diabetes status $(n=8$; Supplementary Table 1$)$, resulting in a final study population of 138 individuals ( $n=133$ for analyses with cognitive performance). Individuals who were excluded because of missing data did not differ from those who were included in the present analyses (Supplementary Table 1).

The Pearl Neurodegenerative Diseases was approved by the Medical Ethics Review Committee of the VU University Medical Centre, and written informed consent was obtained from patients enrolled in the dataset.

\section{Cerebrospinal fluid measures}

Lumbar puncture was performed at the L3/L4 or L4/L5 intervertebral space and $3 \mathrm{ml}$ of the collected CSF was aliquoted in $0.5 \mathrm{ml}$ samples, which were stored at $-80^{\circ} \mathrm{C}$ [27]. From these samples, $A \beta_{1-42}$, Tau, and p-Tau were measured using Innotest $^{\circledR}$ Enzyme-Linked Immunosorbent Assays (Innogenetics, Ghent, Belgium) [27]. These assays were performed at the Neurochemistry Laboratory of the department of Clinical Chemistry of the VU Medical Centre Amsterdam according to described procedures [27]. The samples were analyzed all at once, using the same batch of reagents.

CSF levels of insulin were measured at the same department, in the Endocrine Laboratory, in the CSF volume that remained after aliquots had been taken for $A \beta_{1-42}$, Tau, and $p$-Tau assays, a leftover volume that ranged between 75 and $150 \mu \mathrm{L}$ per patient. CSF insulin levels were measured using an ultrasensitive radioimmunoassay (EMD Millipore, Bellerica, $\mathrm{MA}$ ) with a detection limit of $1.2 \mathrm{pmol} / \mathrm{L}$. The limited amount of CSF available prevented us from measuring insulin in duplicate. Note that patients were not required to fast prior to lumbar puncture.

\section{Cognitive performance}

As part of the diagnostic workup, patients completed a standardized battery of cognitive tests [27]. We selected neuropsychological tests that reflect global cognition (i.e., MMSE [31], a screening test) and memory performance (i.e., AVLT [28, 29]), the latter because insulin signaling has been primarily related to memory performance [32,33]. Total immediate and delayed recall scores of the ALVT were combined into one composite memory score. To this end, raw test scores were transformed into z-scores and averaged.

\section{Covariates}

A detailed description of the covariates (i.e., educational level, medical history, and lifestyle factors) is provided in the Supplementary Material.

\section{Statistical analyses}

Differences in demographic and clinical characteristics across the diagnostic groups (i.e., SCI, aMCI, and $\mathrm{AD}$ ) were evaluated using analysis of variance for continuous variables and chi-square tests for categorical variables. Because there are currently limited data on variables that determine CSF levels of insulin, we used univariate linear regression to explore the association between CSF insulin and the available set of demographic and clinical variables known to be associated with cognitive performance (i.e., age, sex, educational level, blood pressure, body mass index, diabetes, cardiovascular or cerebrovascular disease, smoking behavior, and alcohol consumption). In these analyses, CSF insulin was taken as the dependent variable and assumptions of linear regression were verified prior to analyses. Variables that 
showed a significant association with CSF insulin ( $p$-value $<0.05)$ were considered as potentially confounding factors in subsequent multivariate analyses.

With use of analysis of covariance, we examined whether CSF insulin levels differed between the diagnostic groups, independent of the identified potentially confounding variables. Multiple linear regression analyses were then conducted to examine the association of CSF insulin with cognitive performance (i.e., MMSE and composite memory score) and CSF biomarkers of $\mathrm{AD}$ (i.e., $\mathrm{A} \beta_{1-42}$, Tau, and p-Tau). In these main analyses, which were performed across and within each diagnostic group, CSF insulin was the independent variable. Two predefined models of adjustment were used (model 1: clinic site; model 2: model $1+$ age and sex). The third model also included those variables being associated with CSF insulin in the univariate analyses.

Multiple interaction terms (i.e., CSF insulin * sex and CSF insulin * APOE $\varepsilon 4$ carriership) were used to explore whether any association between CSF insulin and cognitive performance or Alzheimer pathology differed by sex or APOE $\varepsilon 4$ genotype. Likewise, we examined whether any association differed by the presence of diabetes given that diabetes is characterized by abnormal insulin signaling, at least in the periphery [34]. In case of significant interaction, stratified analyses were performed. We additionally evaluated whether any of the univariate associations observed between CSF insulin and demographic or clinical characteristics differed by sex or APOE $\varepsilon 4$ genotype.

As a sensitivity analysis, we reanalyzed the data with exclusion of individuals previously diagnosed with diabetes.

All analyses were performed with use of SPSS for Windows, version 23.0 (IBM SPSS, IBM Corp, Armonk, NY, USA). Levels of CSF insulin, Tau, and p-Tau were transformed with the natural logarithm prior to the analyses. A two-sided $p$-value $<0.05$ was considered significant, except for interaction analyses where the significance level was set at 0.10 to compensate for the loss of statistical power in these analyses [35]. No adjustments were made for multiple testing [36].

\section{RESULTS}

Table 1 shows the demographic and clinical characteristics of the study population as a whole, by sex, and by diagnostic group [i.e., SCI $(n=45)$; aMCI $(n=44)$, and $\mathrm{AD}(n=49)]$. Overall, patients were in late adulthood ( $66 \pm 9$ years), with a slight male predominance $(65.2 \%)$. Men had somewhat unhealthier lifestyle variables than women and more frequently reported a history of cardiovascular disease. Cerebrovascular disease was, however, equally distributed between the sexes. Of note, the cardiovascular risk profile was similar across the diagnostic groups, except for the presence of diabetes, which was least common in the SCI group.

\section{CSF insulin in relation to demographic and clinical variables: Basic findings}

Table 2 shows the univariate associations of clinical and demographic variables with CSF levels of insulin in the total study population. Diabetes status and higher body mass index were associated with higher CSF insulin levels, whereas carriers of the APOE $\varepsilon 4$ allele had lower CSF insulin in comparison with non-carriers There were no significant associations with the other variables.

Multiplicative interaction analyses showed that sex modified the univariate association between diabetes and CSF insulin in that diabetes was more strongly associated with higher CSF insulin levels in men than women. Sex also modified the association between APOE $\varepsilon 4$ and CSF insulin. Specifically, APOE $\varepsilon 4$ carriership was more strongly associated with lower CSF insulin levels in women than men, although the association between APOE $\varepsilon 4$ and CSF reached statistical significance in neither of the sexes. APOE $\varepsilon 4$ carriership itself, in turn, modified the univariate association between diabetes and CSF insulin in that diabetes was only associated with higher CSF insulin levels in non-carriers of the APOE $\varepsilon 4$ allele. Please note that CSF insulin levels did not differ between sexes and diabetes was not more common among men (Table 1). Likewise, APOE $\varepsilon 4$ genotype did not differ between sexes (Table 1).

\section{CSF insulin in the diagnostic groups of cognitive impairment}

Median (interquartile range) levels of CSF insulin in individuals with SCI, aMCI, and AD were $3.77 \mathrm{pmol} / \mathrm{L}$ (3.34-4.39), 3.61 pmol/L (3.22-4.36), and $3.98 \mathrm{pmol} / \mathrm{L}$ (3.57-4.77), respectively. Despite the seemingly higher levels of CSF insulin in AD, CSF insulin did not differ significantly between the groups $(p=0.136)$. The linear trend across the 
Table 1

Demographic and clinical characteristics of the study population as a whole and stratified by sex and diagnostic group

\begin{tabular}{|c|c|c|c|c|c|c|c|c|}
\hline & $\begin{array}{c}\text { Total } \\
(n=138)\end{array}$ & $\begin{array}{c}\text { Men } \\
(n=90)\end{array}$ & $\begin{array}{l}\text { Women } \\
(n=48)\end{array}$ & $\begin{array}{c}p \text {-value for } \\
\text { difference }\end{array}$ & $\begin{array}{c}\mathrm{SCI} \\
(n=45)\end{array}$ & $\begin{array}{c}\mathrm{aMCI} \\
(n=44)\end{array}$ & $\begin{array}{c}\mathrm{AD} \\
(n=49)\end{array}$ & $\begin{array}{l}p \text {-value for } \\
\text { trend }\end{array}$ \\
\hline Age (y) & $66 \pm 9$ & $66 \pm 9$ & $65 \pm 10$ & 0.564 & $62 \pm 9$ & $67 \pm 9$ & $68 \pm 9$ & $<0.001$ \\
\hline Male & $90(65.2 \%)$ & $\ldots$ & $\ldots$ & $\ldots$ & $30(66.7 \%)$ & $30(68.2 \%)$ & $30(61.2 \%)$ & 0.573 \\
\hline \multicolumn{9}{|l|}{ Educational level } \\
\hline Low & $32(23.2 \%)$ & $21(23.3 \%)$ & $11(22.9 \%)$ & & $10(22.2 \%)$ & $11(25.0 \%)$ & $11(22.4 \%)$ & \\
\hline Middle & $52(37.7 \%)$ & $27(30.0 \%)$ & $25(52.1 \%)$ & 0.020 & $16(35.6 \%)$ & $19(43.2 \%)$ & $17(34.7 \%)$ & 0.962 \\
\hline High & $54(39.1 \%)$ & $42(46.7 \%)$ & $12(25.0 \%)$ & & $19(42.2 \%)$ & $14(31.8 \%)$ & $21(42.9 \%)$ & \\
\hline $\mathrm{SBP}(\mathrm{mmHg})^{*}$ & $145 \pm 19$ & $145 \pm 19$ & $146 \pm 20$ & 0.933 & $142 \pm 19$ & $145 \pm 17$ & $149 \pm 22$ & 0.127 \\
\hline $\mathrm{DBP}(\mathrm{mmHg})^{*}$ & $84 \pm 10$ & $85 \pm 10$ & $83 \pm 10$ & 0.325 & $83 \pm 11$ & $84 \pm 7$ & $84 \pm 12$ & 0.470 \\
\hline BMI $\left(\mathrm{kg} / \mathrm{m}^{2}\right)$ & $25.2 \pm 3.2$ & $25.6 \pm 3.2$ & $24.7 \pm 3.1$ & 0.113 & $25.7 \pm 3.2$ & $25.3 \pm 3.0$ & $24.8 \pm 3.4$ & 0.194 \\
\hline Diabetes & $16(11.6 \%)$ & $12(13.3 \%)$ & $4(8.3 \%)$ & 0.382 & $2(4.4 \%)$ & $5(11.4 \%)$ & $9(18.4 \%)$ & 0.036 \\
\hline Cardiovascular disease & $43(31.2 \%)$ & $38(42.2 \%)$ & $5(10.4 \%)$ & $<0.001$ & $17(37.8 \%)$ & $13(29.5 \%)$ & $13(26.5 \%)$ & 0.244 \\
\hline Cerebrovascular disease & $17(12.3 \%)$ & $12(13.3 \%)$ & $5(10.4 \%)$ & 0.620 & $7(15.6 \%)$ & $3(6.8 \%)$ & $7(14.3 \%)$ & 0.877 \\
\hline APOE $\varepsilon 4$ carrier & $81(58.7 \%)$ & $52(57.8 \%)$ & $29(60.4 \%)$ & 0.766 & $20(44.4 \%)$ & $31(70.5 \%)$ & $30(61.2 \%)$ & 0.110 \\
\hline \multicolumn{9}{|l|}{ Smoking behavior* } \\
\hline Never & $58(43.0 \%)$ & $29(32.2 \%)$ & $29(60.4 \%)$ & & $16(35.6 \%)$ & $19(45.2 \%)$ & $23(47.9 \%)$ & \\
\hline Former & $56(41.5 \%)$ & $42(46.7 \%)$ & $14(29.2 \%)$ & 0.005 & $23(51.1 \%)$ & $15(35.7 \%)$ & $18(37.5 \%)$ & 0.454 \\
\hline Current & $21(15.6 \%)$ & $17(18.9 \%)$ & $4(8.3 \%)$ & & $6(13.3 \%)$ & $8(19.0 \%)$ & $7(14.6 \%)$ & \\
\hline Alcohol consumption* & $110(80.3 \%)$ & $78(86.7 \%)$ & $32(66.7 \%)$ & 0.003 & $37(82.2 \%)$ & $35(81.4 \%)$ & $38(77.6 \%)$ & 0.568 \\
\hline CSF insulin $(\mathrm{pmol} / \mathrm{L})$ & $3.79[3.37-4.52]$ & $3.85[3.43-4.60]$ & $3.65[3.12-4.40]$ & 0.234 & $3.77[3.34-4.39]$ & $3.61[3.22-4.36]$ & $3.98[3.57-4.77]$ & 0.059 \\
\hline $\mathrm{CSF} A \beta_{1-42}(\mathrm{ng} / \mathrm{L})$ & $678 \pm 281$ & $701 \pm 301$ & $636 \pm 237$ & 0.172 & $835 \pm 244$ & $656 \pm 279$ & $554 \pm 250$ & $<0.001$ \\
\hline CSF Tau (ng/L) & 421 [229-633] & 397 [231-619] & 494 [222-707] & 0.418 & $229[176-354]$ & $485[267-651]$ & $544[401-864]$ & $<0.001$ \\
\hline CSF p-Tau (ng/L) & $55[36-77]$ & $54[36-76]$ & $57[39-86]$ & 0.663 & $36[23-55]$ & $58[40-83]$ & $71[53-89]$ & $<0.001$ \\
\hline MMSE (score)* & $26 \pm 3$ & $27 \pm 3$ & $26 \pm 3$ & 0.111 & $28 \pm 2$ & $27 \pm 3$ & $24 \pm 3$ & $<0.001$ \\
\hline \multicolumn{9}{|l|}{ AVLT (words) } \\
\hline Total immediate recall* & $30 \pm 11$ & $30 \pm 11$ & $31 \pm 13$ & 0.408 & $41 \pm 10$ & $26 \pm 7$ & $25 \pm 8$ & $<0.001$ \\
\hline Delayed recall* & $4 \pm 4$ & $4 \pm 3$ & $5 \pm 4$ & 0.418 & $8 \pm 3$ & $2 \pm 2$ & $3 \pm 3$ & $<0.001$ \\
\hline
\end{tabular}

Data are presented as $\mathrm{n}(\%)$, mean $\pm \mathrm{SD}$, or median [IQR]. * Data not available from all participants $(n=137$ for alcohol consumption; $n=136$ for AVLT total immediate recall; $n=135$ for SBP, DBP, smoking behavior, MMSE, and AVLT delayed recall). SCI, subjective cognitive impairment; aMCI, amnestic mild cognitive impairment; AD, Alzheimer's dementia; SBP, systolic blood pressure; DBP, diastolic blood pressure; BMI, body mass index; CSF, cerebrospinal fluid; MMSE, Mini-Mental State Examination; AVLT, 15 Word-Auditory Verbal Learning Test. 
Table 2

Univariate associations of clinical and demographic characteristics with CSF insulin levels in the whole study population and stratified according to sex and APOE $\varepsilon 4$ genotype where applicable

\begin{tabular}{|c|c|c|c|c|c|c|}
\hline & $\begin{array}{c}n \\
\left(\mathrm{n}_{\max }=138\right)\end{array}$ & $\beta$ & $\begin{array}{c}p \text {-value for } \\
\text { interaction } \\
\text { with sex }\end{array}$ & $\beta$ & $\begin{array}{c}p \text {-value for } \\
\text { interaction with } \\
\text { APOE } \varepsilon 4 \text { carriership }\end{array}$ & \\
\hline$\overline{\text { Age }}$ & 138 & $0.012(-0.158 ; 0.181)$ & 0.315 & $\ldots$ & 0.792 & $\ldots$ \\
\hline Sex & & & & & & \\
\hline male versus female & 138 & $0.214(-0.139 ; 0.566)$ & $\ldots$ & $\ldots$ & 0.540 & $\ldots$ \\
\hline Educational level & & & & & & \\
\hline medium versus low & 138 & $-0.253(-0.698 ; 0.193)$ & 0.886 & $\ldots$ & 0.904 & $\ldots$ \\
\hline high versus medium & & $0.068(-0.317 ; 0.453)$ & 0.477 & $\ldots$ & 0.432 & $\ldots$ \\
\hline high versus low & & $-0.185(-0.627 ; 0.258)$ & 0.453 & $\ldots$ & 0.569 & $\ldots$ \\
\hline SBP & 135 & $0.101(-0.070 ; 0.271)$ & 0.240 & $\ldots$ & 0.562 & $\ldots$ \\
\hline DBP & 135 & $0.039(-0.132 ; 0.211)$ & 0.369 & $\ldots$ & 0779 & $\ldots$ \\
\hline BMI & 138 & $0.261(0.097 ; 0.425)$ & 0.756 & $\ldots$ & 0.326 & $\ldots$ \\
\hline $\begin{array}{l}\text { Diabetes } \\
\text { yes versus no }\end{array}$ & 138 & $1.152(0.662 ; 1.642)$ & $<0.001$ & $\begin{array}{r}\text { Men: } 2.232(1.401 ; 3.063) \\
\text { Women: } 0.657(0.053 ; 1.261)\end{array}$ & 0.012 & $\begin{array}{c}\text { Carriers: } 0.528(-0.230 ; 1.285) \\
\text { Non-carriers: } 1.706(1.065 ; 2.346)\end{array}$ \\
\hline $\begin{array}{l}\text { Cardiovascular disease } \\
\text { yes versus no }\end{array}$ & 138 & $0.211(-0.153 ; 0.574)$ & 0.492 & $\ldots$ & 0.704 & $\ldots$ \\
\hline $\begin{array}{l}\text { Cerebrovascular disease } \\
\text { yes versus no }\end{array}$ & 138 & $0.089(-0.425 ; 0.603)$ & 0.360 & & 0.784 & $\ldots$ \\
\hline $\begin{array}{l}\text { APOE } \varepsilon 4 \text { genotype } \\
\text { carriers versus non-carriers }\end{array}$ & 138 & $-0.547(-1.078 ;-0.016)$ & 0.092 & $\begin{array}{r}\text { Men: }-0.192(-0.698 ; 0.313) \\
\text { Women: }-1.118(-2.295 ; 0.059)\end{array}$ & $\ldots$ & $\ldots$ \\
\hline $\begin{array}{l}\text { Smoking behavior } \\
\text { former versus never } \\
\text { current versus former } \\
\text { current versus never }\end{array}$ & 135 & $\begin{array}{r}0.121(-0.252 ; 0.494) \\
-0.025(-0.534 ; 0.484) \\
0.096(-0.410 ; 0.603)\end{array}$ & $\begin{array}{l}0.455 \\
0.319 \\
0.615\end{array}$ & $\begin{array}{l}\cdots \\
\cdots \\
\cdots\end{array}$ & $\begin{array}{l}0.400 \\
0.519 \\
0.520\end{array}$ & $\begin{array}{l}\cdots \\
\cdots \\
\cdots\end{array}$ \\
\hline $\begin{array}{l}\text { Alcohol consumption } \\
\text { yes versus no }\end{array}$ & 137 & $-0.080(-0.506 ; 0.347)$ & 0.650 & .. & 0.090 & $\begin{array}{c}\text { Carriers: } 0.395(-0.301 ; 1.091) \\
\text { Non-carriers: }-0.365(-0.910 ; 0.179)\end{array}$ \\
\hline
\end{tabular}

Data are presented as standardized regression coefficients $\beta$ (95\% confidence intervals) for continuous variables and between-group differences (95\% confidence intervals) for categorical variables, the latter expressed in standard deviations of cerebrospinal fluid insulin levels. $p$-values are derived from interaction analyses with sex and APOE $\varepsilon 4$ carriership. Insulin levels were transformed with the natural logarithm prior to analysis. CSF, cerebrospinal fluid; SBP, systolic blood pressure; DBP, diastolic blood pressure; BMI, body mass index. 
Table 3

Association of CSF insulin levels with cognitive performance

\begin{tabular}{|c|c|c|c|c|c|c|}
\hline & $\begin{array}{c}\text { Total } \\
(n=133)\end{array}$ & $\begin{array}{c}\text { SCI } \\
(n=43)\end{array}$ & $\begin{array}{c}\text { aMCI } \\
(n=44)\end{array}$ & $\begin{array}{c}\mathrm{AD} \\
(n=46)\end{array}$ & $\begin{array}{c}\text { Men } \\
(n=86)\end{array}$ & $\begin{array}{l}\text { Women } \\
(n=47)\end{array}$ \\
\hline \multicolumn{7}{|l|}{ MMSE } \\
\hline Model 1 & $\begin{array}{c}-0.023 \\
(-0.196 ; 0.150)\end{array}$ & $\begin{array}{c}0.084 \\
(-0.238 ; 0.405)\end{array}$ & $\begin{array}{c}0.103 \\
(-0.203 ; 0.410)\end{array}$ & $\begin{array}{c}0.021 \\
(-0.288 ; 0.329)\end{array}$ & $\begin{array}{c}0.144 \\
(-0.072 ; 0.360)\end{array}$ & $\begin{array}{c}-0.242 \\
(-0.537 ; 0.053)\end{array}$ \\
\hline Model 2 & $\begin{array}{c}-0.045 \\
(-0.209 ; 0.120)\end{array}$ & $\begin{array}{c}0.111 \\
(-0.231 ; 0.454)\end{array}$ & $\begin{array}{c}0.095 \\
(-0.200 ; 0.390)\end{array}$ & $\begin{array}{c}-0.017 \\
(-0.322 ; 0.288)\end{array}$ & $\begin{array}{c}0.121 \\
(-0.092 ; 0.333)\end{array}$ & $\begin{array}{c}-0.210 \\
(-0.481 ; 0.061)\end{array}$ \\
\hline Model 3 & $\begin{array}{c}-0.071 \\
(-0.252 ; 0.111)\end{array}$ & $\begin{array}{c}0.175 \\
(-0.190 ; 0.540)\end{array}$ & $\begin{array}{c}0.045 \\
(-0.323 ; 0.413)\end{array}$ & $\begin{array}{c}-0.014 \\
(-0.352 ; 0.324)\end{array}$ & $\begin{array}{c}0.123 \\
(-0.108 ; 0.354)\end{array}$ & $\begin{array}{c}-0.483^{*} \\
(-0.831 ;-0.135)\end{array}$ \\
\hline \multicolumn{7}{|l|}{ Composite memory score } \\
\hline Model 1 & $\begin{array}{c}-0.051 \\
(-0.224 ; 0.122)\end{array}$ & $\begin{array}{c}-0.324^{*} \\
(-0.619 ;-0.030)\end{array}$ & $\begin{array}{c}0.164 \\
(-0.143 ; 0.472)\end{array}$ & $\begin{array}{c}0.210 \\
(-0.091 ; 0.511)\end{array}$ & $\begin{array}{c}0.120 \\
(-0.085 ; 0.324)\end{array}$ & $\begin{array}{c}-0.206 \\
(-0.489 ; 0.078)\end{array}$ \\
\hline Model 2 & $\begin{array}{c}-0.048 \\
(-0.206 ; 0.109)\end{array}$ & $\begin{array}{c}-0.251 \\
(-0.549 ; 0.047)\end{array}$ & $\begin{array}{c}0.170 \\
(-0.103 ; 0.444)\end{array}$ & $\begin{array}{c}0.162 \\
(-0.143 ; 0.467)\end{array}$ & $\begin{array}{c}0.078 \\
(-0.108 ; 0.264)\end{array}$ & $\begin{array}{c}-0.174 \\
(-0.434 ; 0.086)\end{array}$ \\
\hline Model 3 & $\begin{array}{c}-0.014 \\
(-0.188 ; 0.159)\end{array}$ & $\begin{array}{c}-0.092 \\
(-0.381 ; 0.197)\end{array}$ & $\begin{array}{c}0.162 \\
(-0.177 ; 0.502)\end{array}$ & $\begin{array}{c}0.251 \\
(-0.078 ; 0.580)\end{array}$ & $\begin{array}{c}0.150 \\
(-0.048 ; 0.347)\end{array}$ & $\begin{array}{c}-0.273 \\
(-0.628 ; 0.081)\end{array}$ \\
\hline
\end{tabular}

Data are presented as standardized regression coefficients, which reflect the standard deviation change in cognitive performance per standard deviation increase in cerebrospinal fluid insulin levels. Insulin levels were transformed with the natural logarithm prior to analysis. Stratified analyses according to sex were performed as interaction analyses revealed statistically significant interaction of this factor on the association between CSF insulin and cognitive performance. ${ }^{*} p<0.05$. Model 1: adjusted for UMC; Model 2: additionally adjusted for age and sex (the latter not in case of stratified analysis according to sex); Model 3: additionally adjusted for BMI and the presence of diabetes. CSF, cerebrospinal fluid; SCI, subjective cognitive impairment; aMCI, amnestic mild cognitive impairment; AD, Alzheimer's disease; UMC, University Medical Center; BMI, body mass index.

diagnostic groups was borderline significant ( $\mathrm{p}$ for trend $=0.059$ ). There were no interactions with sex or APOE $\varepsilon 4$ genotype ( $\mathrm{p}$ for interaction $=0.707$ and 0.120 , respectively). When diabetes status and body mass index were included as covariates, pairwise comparisons between the diagnostic groups confirmed that CSF insulin did not differ significantly between the groups (all $p>0.105$ ).

\section{CSF insulin and cognitive performance}

Table 3 shows the association of CSF insulin with cognitive performance across the groups and within each diagnostic group. After adjustment for center, CSF insulin was not associated with cognitive performance in the overall study population, nor within the groups of individuals with aMCI or AD. Among individuals with SCI, higher CSF insulin levels were associated with worse memory function, but this association lost significance once adjustments were made for age and sex.

Multiplicative interaction analyses revealed that the null associations between CSF insulin and cognitive performance did not differ by the presence of diabetes (all $\mathrm{p}$ for interaction $>0.614$ ) or APOE $\varepsilon 4$ genotype (all $p>0.425$ ), but did differ by sex (all $p<0.059$ ). Subsequent stratified analyses (Table 3, right panel) showed that among women, but not among men, higher CSF insulin were associated with worse cognitive performance. These associations were significant for MMSE performance in the fully adjusted model.

\section{CSF insulin and CSF biomarkers of $A D$}

Table 4 shows the associations of CSF insulin with CSF biomarkers of $\mathrm{AD}$ across and within each diagnostic group. In brief, CSF insulin was not associated with CSF $A \beta_{1-42}$, nor with levels of Tau or $p$-Tau.

Multiplicative interaction analyses showed that diabetes did not modify these null associations (all $\mathrm{p}$ for interaction $>0.170$ ), while sex and APOE $\varepsilon 4$ genotype did, at least for the association between CSF insulin and (p-)Tau (all $p<0.017$ and $<0.096$, respectively). Subsequent stratified analyses (Table 4 , right panel) showed that among women, but not among men, higher CSF insulin levels were associated with higher levels of tau and tended to be associated with higher levels of p-Tau in the fully adjusted model. In $\mathrm{APOE} \varepsilon 4$ non-carriers, but not in carriers, higher CSF insulin was associated with both higher tau and p-Tau levels in the fully adjusted model. Notably, associations were strongest among female patients who were non-carriers of the APOE $\varepsilon 4$ allele (data not shown).

\section{Sensitivity analysis}

When the data were reanalyzed excluding individuals with diabetes, qualitatively similar results were obtained (Supplementary Tables 2 and 3). 
Table 4

Associations of CSF insulin levels with levels of CSF A $\beta_{1-42}$ and (p-)Tau

\begin{tabular}{|c|c|c|c|c|c|c|c|c|}
\hline & $\begin{array}{c}\text { Total } \\
(n=138)\end{array}$ & $\begin{array}{c}\text { SCI } \\
(n=45)\end{array}$ & $\begin{array}{c}\mathrm{aMCI} \\
(n=44)\end{array}$ & $\begin{array}{c}\mathrm{AD} \\
(n=49)\end{array}$ & $\begin{array}{c}\text { Men } \\
(n=90)\end{array}$ & $\begin{array}{l}\text { Women } \\
(n=48)\end{array}$ & $\begin{array}{c}\text { APOE } \varepsilon 4 \\
\text { carriers } \\
(n=81)\end{array}$ & $\begin{array}{c}\text { APOE } \varepsilon 4 \\
\text { non-carriers } \\
(n=57)\end{array}$ \\
\hline \multicolumn{9}{|l|}{$\mathrm{CSF} A \beta_{1-42}$} \\
\hline Model 1 & $\begin{array}{c}-0.013 \\
(-0.183 ; 0.157)\end{array}$ & $\begin{array}{c}-0.006 \\
(-0.320 ; 0.309)\end{array}$ & $\begin{array}{c}0.057 \\
(-0.258 ; 0.373)\end{array}$ & $\begin{array}{c}0.118 \\
(-0.167 ; 0.403)\end{array}$ & $\ldots$ & $\ldots$ & $\ldots$ & $\ldots$ \\
\hline Model 2 & $\begin{array}{c}-0.023 \\
(-.185 ; 0.139)\end{array}$ & $\begin{array}{c}-0.034 \\
(-0.354 ; 0.285)\end{array}$ & $\begin{array}{c}0.061 \\
(-0.245 ; 0.366)\end{array}$ & $\begin{array}{c}0.087 \\
(-0.204 ; 0.378)\end{array}$ & $\ldots$ & $\ldots$ & . & $\ldots$ \\
\hline Model 3 & $\begin{array}{c}-0.130 \\
(-0.303 ; 0.043)\end{array}$ & $\begin{array}{c}-0.001 \\
(-0.355 ; 0.353)\end{array}$ & $\begin{array}{c}-0.185 \\
(-0.519 ; 0.149)\end{array}$ & $\begin{array}{c}-0.059 \\
(-0.362 ; 0.245)\end{array}$ & $\ldots$ & $\ldots$ & . & $\ldots$ \\
\hline \multicolumn{9}{|l|}{ CSF Tau } \\
\hline Model 1 & $\begin{array}{c}0.006 \\
(-0.164 ; 0.177)\end{array}$ & $\begin{array}{c}0.171 \\
(-0.139 ; 0.481)\end{array}$ & $\begin{array}{c}-0.200 \\
(-0.495 ; 0.096)\end{array}$ & $\begin{array}{c}-0.119 \\
(-0.414 ; 0.177)\end{array}$ & $\begin{array}{c}-0.184 \\
(-0.394 ; 0.026)\end{array}$ & $\begin{array}{c}0.236 \\
(-0.055 ; 0.528)\end{array}$ & $\begin{array}{c}-0.147 \\
(-0.370 ; 0.077)\end{array}$ & $\begin{array}{c}0.253 \\
(-0007 ; 0.513)\end{array}$ \\
\hline Model 2 & $\begin{array}{c}0.012 \\
(-0.140 ; 0.164)\end{array}$ & $\begin{array}{c}0.097 \\
(-0.165 ; 0.359)\end{array}$ & $\begin{array}{c}-0.202 \\
(-0.466 ; 0.062)\end{array}$ & $\begin{array}{c}-0.114 \\
(-0.412 ; 0.185)\end{array}$ & $\begin{array}{c}-0.147 \\
(-0.335 ; 0.040)\end{array}$ & $\begin{array}{c}0.194 \\
(-0.071 ; 0.459)\end{array}$ & $\begin{array}{c}-0.137 \\
(-0.347 ; 0.074)\end{array}$ & $\begin{array}{c}0.232^{*} \\
(0.014 ; 0.450)\end{array}$ \\
\hline Model 3 & $\begin{array}{c}0.059 \\
(-0.104 ; 0.222)\end{array}$ & $\begin{array}{c}0.189 \\
(-0.089 ; 0.467)\end{array}$ & $\begin{array}{c}-0.060 \\
(-0.361 ; 0.241)\end{array}$ & $\begin{array}{c}-0.133 \\
(-0.462 ; 0.196)\end{array}$ & $\begin{array}{c}-0.098 \\
(-0.297 ; 0.101)\end{array}$ & $\begin{array}{c}0.311 \\
(-0.030 ; 0.653)\end{array}$ & $\begin{array}{c}-0.189 \\
(-0.441 ; 0.063)\end{array}$ & $\begin{array}{c}0.287^{*} \\
(0.079 ; 0.495)\end{array}$ \\
\hline \multicolumn{9}{|l|}{ CSF p-Tau } \\
\hline Model 1 & $\begin{array}{c}0.037 \\
(-0.131 ; 0.206)\end{array}$ & $\begin{array}{c}0.165 \\
(-0.140 ; 0.471)\end{array}$ & $\begin{array}{c}-0.162 \\
(-0.473 ; 0.150)\end{array}$ & $\begin{array}{c}-0.067 \\
(-0.358 ; 0.225)\end{array}$ & $\begin{array}{c}-0.174 \\
(-0.383 ; 0.035)\end{array}$ & $\begin{array}{c}0.287^{*} \\
(0.003 ; 0.571)\end{array}$ & $\begin{array}{c}-0.073 \\
(-0.294 ; 0.148)\end{array}$ & $\begin{array}{c}0.224 \\
(-0.042 ; 0.490)\end{array}$ \\
\hline Model 2 & $\begin{array}{c}0.039 \\
(-0.116 ; 0.195)\end{array}$ & $\begin{array}{c}0.097 \\
(-0.184 ; 0.377)\end{array}$ & $\begin{array}{c}-0.164 \\
(-0.455 ; 0.127)\end{array}$ & $\begin{array}{c}-0.069 \\
(-0.369 ; 0.231)\end{array}$ & $\begin{array}{c}-0.145 \\
(-0.340 ; 0.051)\end{array}$ & $\begin{array}{c}0.246 \\
(-0.013 ; 0.505)\end{array}$ & $\begin{array}{c}-0.066 \\
(-0.282 ; 0.150)\end{array}$ & $\begin{array}{c}0.201 \\
(-0.028 ; 0.430)\end{array}$ \\
\hline Model 3 & $\begin{array}{c}0.074 \\
(-0.090 ; 0.239) \\
\end{array}$ & $\begin{array}{c}0.207 \\
(-0.082 ; 0.497) \\
\end{array}$ & $\begin{array}{c}-0.036 \\
(-0.370 ; 0.298) \\
\end{array}$ & $\begin{array}{c}-0.100 \\
(-0.426 ; 0.225) \\
\end{array}$ & $\begin{array}{c}-0.088 \\
(-0.290 ; 0.113) \\
\end{array}$ & $\begin{array}{c}0.353^{*} \\
(0.018 ; 0.689) \\
\end{array}$ & $\begin{array}{c}-0.105 \\
(-0.360 ; 0.149) \\
\end{array}$ & $\begin{array}{c}0.246^{*} \\
(0.026 ; 0.467) \\
\end{array}$ \\
\hline
\end{tabular}




\section{DISCUSSION}

The present study shows that, among women and non-carriers of the APOE $\varepsilon 4$ allele, higher levels of CSF insulin were associated with worse cognitive performance and/or higher levels of CSF (p-)Tau. CSF insulin did, however, not differ between diagnostic groups of cognitive impairment (i.e., SCI, aMCI, and $\mathrm{AD}$ ). These findings add to the growing body of evidence indicating that abnormal insulin signaling is involved in $\mathrm{AD} \mathrm{[1-4]} \mathrm{and} \mathrm{also} \mathrm{provide} \mathrm{an} \mathrm{explana-}$ tion for why women and non-carriers of the APOE $\varepsilon 4$ allele seem to benefit most from intranasal insulin administration to enhance cognitive performance (in AD) [24-26].

There are few earlier studies on the association between CSF insulin and AD [17-21]. Results on CSF insulin levels in patients with AD were inconsistent and reported CSF insulin to be increased [17], decreased $[18,20]$, or unchanged $[19,21]$ in individuals with $\mathrm{AD}$ as compared to healthy individuals [17-19, 21] or those with SCI [20]. The most probable explanation for these variable results across studies is the use of different study populations. For example, studies reporting decreased levels of CSF insulin in $\operatorname{AD}[18,20]$ excluded individuals with diabetes, while the present study provides evidence that the presence of diabetes is associated with higher levels of CSF insulin. Other differences between the populations concern the severity of the dementia and the use of different reference groups.

Substantially more research has been conducted on the association between cerebral glucose metabolism, based on fluorodeoxyglucose positron emission tomography (FDG-PET) [36-42] or the CSF to plasma glucose ratio [43], and $\mathrm{AD}$ pathology. Collectively, these studies suggest that cerebral hypometabolism is more prominent in individuals with $\mathrm{AD}$ [42] and is associated with higher CSF (p-)Tau [36-39] and lower CSF A $\beta_{1-42}[36,38,40$, $41,43]$ levels. Although it has been suggested that cerebral hypometabolism may reflect cerebral insulin resistance [43], it is important to realize that glucose uptake in the brain is largely independent of insulin levels [44] Even in the hippocampus, one of the brain regions with the highest concentrations of insulin sensitive GLUT-4 receptors, it is believed that glucose update does not necessary require insulin [44]. The link between cerebral hypometabolism and AD does, thus, not directly support a role for insulin resistance in AD pathology. This is particularly the case because glucose itself may have a direct effect on tau metabolism [45] and because it has been suggested that tau-related pathology may precede cerebral hypometabolism rather than being a consequence of it [46].

Despite the numerous reviews that have been published on the mechanisms through which cerebral insulin might interfere with $\mathrm{A} \beta$ and tau metabolism (e.g., [4, 47]), only one previous study has actually evaluated CSF insulin in relation to CSF $A \beta_{1-42}$ and (p-)Tau levels [20]. The overall null results of that study are in line with the null associations we observed in our overall study population. We, however, now show that higher levels of CSF insulin were associated with higher levels of CSF (p-)Tau in women and non-carriers of the APOE $\varepsilon 4$ allele, which reinforces data from experimental studies linking insulin to tau metabolism. From a pathophysiological viewpoint, higher levels of CSF insulin, potentially reflecting cerebral insulin resistance and thus deficient brain insulin signaling, may result in reduced activity of the phosphoinositide 3-kinaseprotein kinase B pathway. This, in turn, leads to over activation of glycogen synthase kinase-3, and consequently, hyperphosphorylation of tau [47]. That the association between CSF insulin and tau differs with APOE $\varepsilon 4$ genotype could be explained by a higher degree of insulin resistance among non-carriers relative to carriers of the APOE $\varepsilon 4$ allele, as illustrated by differences in insulin-mediated glucose disposal rates [16]. Alternatively, a potential association between CSF insulin and tau in APOE $\varepsilon 4$ carriers might have been simply overshadowed by the high AD risk associated with APOE $\varepsilon 4$ carriership.

The sex differences observed in our study are in line with experimental data and clinical studies showing that the brains of men and women are differentially sensitive to the effects of insulin [23, 24, 48, 49]. More specifically, previous studies have shown that the anorexigenic effects of intranasally administered insulin are more prominent in men, while its memory enhancing effects are more prominent women. It has been suggested [24] that these sex differences can be explained by the cognitive effects of estrogen [50]. A more recent study, however, showed that young and postmenopausal women are comparable sensitivity to the memory enhancing effects of intranasal insulin [51], which reiterates that estrogen explains the observed sex differences. What explains these differences should be explored in future studies. Perhaps it may relate to other physiological or anatomical factors that differ between sexes, for example the density of hippocampal insulin receptors. 
Notably, we did not observe any association between CSF levels of insulin and $\mathrm{A} \beta$, while a previous study noted that a single dose of intranasal insulin increased plasma levels of the short form of $A \beta$ (i.e., $A \beta_{40}$ ) [52]. A potential explanation for this finding are the complex and dual effects insulin (signaling) may have on $A \beta$ clearance [4]. On the one hand, insulin may promote $A \beta$ clearance by accelerating its intraneuronal transport and increasing protein levels of the insulin degrading enzyme (IDE), a metalloprotease that is able to degrade $A \beta$ [4]. On the other hand, IDE also catabolizes insulin, for which it has a higher affinity, which means that insulin at the same time can inhibit $A \beta$ degradation [4]. An alternative explanation is that cerebral insulin is involved in $\mathrm{AD}$ via pathways other than $A \beta$ metabolism (reviewed in [33]).

The present study has limitations. First and foremost, CSF samples were not necessarily taken in the fasting state, nor was lumbar puncture performed at a fixed time of the day. The time of day at which lumbar puncture was performed was also not recorded in the database. Hence, we were unable to adjust our analyses for potential biorhythm effects, while the production and secretion of at least insulin [53] and $A \beta$ [54] seem to follow a circadian rhythm. Although this may have led us to underestimate the association of CSF insulin with AD pathology, it is unlikely to explain the observed modulating effects of either sex or APOE $\varepsilon 4$ genotype. A second limitation that may have further affected our estimates of the association between CSF insulin and CSF pTau concerns the use of an antibody for $\mathrm{p}$-Tau that was specific for phosphorylation at threonine 181, while deficient insulin signaling leads to a widespread phosphorylation of tau at multiple sites. Next, it can be questioned whether higher CSF insulin levels reflect cerebral insulin resistance the way hyperinsulinemia in the plasma reflects peripheral insulin resistance. Note, however, that individuals with diabetes, who are the most likely to have cerebral insulin resistance despite the fact that cerebral and peripheral resistance not necessarily concur [13], had higher levels of CSF insulin. Fourth, multiple analyses were performed without correction for multiple testing, though stratified analyses were only performed in case of statistically significant interaction with a clear a priori hypothesis. Fifth, in the current study, no blood samples were analyzed. Such data would have provided valuable information on how peripheral and cerebral insulin metabolism may interact in relation to cognitive performance and $\mathrm{AD}$ pathology, although compartmentalization is also possible. Finally, it can be questioned whether the composition of the CSF is directly comparable to that of the brain's interstitial fluid [51]. Undoubtedly, however, CSF insulin is a better indicator of deficient brain signaling than blood-derived markers.

In conclusion, our results indicate that higher CSF insulin levels are related to impairment in cognitive performance and biomarkers of $\mathrm{AD}$ among women and non-carriers of the APOE $\varepsilon 4$ allele. As such, our data suggest that the potential involvement of cerebral insulin (resistance) in the pathogenesis of AD is not uniform across individuals. Future studies are needed to determine whether endogenous high CSF insulin levels indeed reflect cerebral insulin resistance, as this might have implications for selection of patients who may benefit from the intranasal use of insulin to improve or preserve cognitive performance. Longitudinal studies are required to strengthen the pathophysiological relevance of our findings.

\section{ACKNOWLEDGMENTS}

The authors would like to thank Paul J. Nederkoorn and Edo Richard for their role as local principal investigators at the AMC University Medical Centre, Amsterdam, the Netherlands. The authors also thank Nico Rozendaal (Maastricht University Medical Centre +) for his role within the Parelsnoer Institute Neurodegenerative Disease. This research was supported by the Parelsnoer Initiative, Parel Neurodegenerative Diseases.

Authors' disclosures available online (http://j-alz. com/manuscript-disclosures/17-0522r1).

\section{SUPPLEMENTARY MATERIAL}

The supplementary material is available in the electronic version of this article: http://dx.doi.org/ 10.3233/JAD-170522.

\section{REFERENCES}

[1] Craft S (2006) Insulin resistance syndrome and Alzheimer disease: Pathophysiologic mechanisms and therapeutic implications. Alzheimer Dis Assoc Disord 20, 298-301.

[2] Schioth HB, Craft S, Brooks SJ, Frey WH 2nd, Benedict C (2012) Brain insulin signaling and Alzheimer's disease: Current evidence and future directions. Mol Neurobiol 46, 4-10.

[3] Cholerton B, Baker LD, Craft S (2013) Insulin, cognition, and dementia. Eur J Pharmacol 719, 170-179. 
[4] Chen Y, Deng Y, Zhang B, Gong CX (2014) Deregulation of brain insulin signaling in Alzheimer's disease. Neurosci Bull 30, 282-294.

[5] Steen E, Terry BM, Rivera EJ, Cannon JL, Neely TR, Tavares R, Xu XJ, Wands JR, de la Monte SM (2005) Impaired insulin and insulin-like growth factor expression and signaling mechanisms in Alzheimer's disease-is this type 3 diabetes? J Alzheimers Dis 7, 63-80.

[6] Ronnemaa E, Zethelius B, Sundelof J, Sundstrom J, Degerman-Gunnarsson M, Berne C, Lannfelt L, Kilander L (2008) Impaired insulin secretion increases the risk of Alzheimer disease. Neurology 71, 1065-1071.

[7] Kuusisto J, Koivisto K, Mykkanen L, Helkala EL, Vanhanen M, Hanninen T, Kervinen K, Kesaniemi YA, Riekkinen PJ, Laakso M (1997) Association between features of the insulin resistance syndrome and Alzheimer's disease independently of apolipoprotein E4 phenotype: Cross sectional population based study. BMJ 315, 1045-1049.

[8] Starks EJ, Patrick O'Grady J, Hoscheidt SM, Racine AM, Carlsson CM, Zetterberg H, Blennow K, Okonkwo OC, Puglielli L, Asthana S, Dowling NM, Gleason CE, Anderson RM, Davenport-Sis NJ, DeRungs LM, Sager MA, Johnson SC, Bendlin BB (2015) Insulin resistance is associated with higher cerebrospinal fluid tau levels in asymptomatic APOE epsilon 4 carriers. J Alzheimers Dis 46, 525-533.

[9] Ekblad LL, Rinne JO, Puukka PJ, Laine HK, Ahtiluoto SE, Sulkava RO, Viitanen MH, Jula AM (2015) Insulin resistance is associated with poorer verbal fluency performance in women. Diabetologia 58, 2545-2553.

[10] Hoscheidt SM, Starks EJ, Oh JM, Zetterberg H, Blennow K, Krause RA, Gleason CE, Puglielli L, Atwood CS, Carlsson CM, Asthana S, Johnson SC, Bendlin BB (2016) Insulin resistance is associated with increased levels of cerebrospinal fluid biomarkers of Alzheimer's Disease and reduced memory function in at-risk healthy middle-aged adults. J Alzheimers Dis 52, 1373-1383.

[11] Schrijvers EM, Witteman JC, Sijbrands EJ, Hofman A, Koudstaal PJ, Breteler MM (2010) Insulin metabolism and the risk of Alzheimer disease: The Rotterdam Study. Neurology 75, 1982-1987.

[12] Luchsinger JA, Tang MX, Shea S, Mayeux R (2004) Hyperinsulinemia and risk of Alzheimer disease. Neurology 63, 1187-1192.

[13] Banks WA, Owen JB, Erickson MA (2012) Insulin in the brain: There and back again. Pharmacol Ther 136, 82-93.

[14] Kern W, Benedict C, Schultes B, Plohr F, Moser A, Born J, Fehm HL, Hallschmid M (2006) Low cerebrospinal fluid insulin levels in obese humans. Diabetologia 49, 27902792.

[15] Plata-Salaman CR (1991) Insulin in the cerebrospinal fluid. Neurosci Biobehav Rev 15, 243-258.

[16] Craft S, Asthana S, Schellenberg G, Cherrier M, Baker LD, Newcomer J, Plymate S, Latendresse S, Petrova A, Raskind M, Peskind E, Lofgreen C, Grimwood K (1999) Insulin metabolism in Alzheimer's disease differs according to apolipoprotein E genotype and gender. Neuroendocrinology 70, 146-152.

[17] Fujisawa Y, Sasaki K, Akiyama K (1991) Increased insulin levels after OGTT load in peripheral blood and cerebrospinal fluid of patients with dementia of Alzheimer type. Biol Psychiatry 30, 1219-1228.

[18] Craft S, Peskind E, Schwartz MW, Schellenberg GD, Raskind M, Porte D Jr (1998) Cerebrospinal fluid and plasma insulin levels in Alzheimer's disease: Relationship to severity of dementia and apolipoprotein E genotype. $\mathrm{Neu}$ rology 50, 164-168.

[19] Molina JA, Jimenez-Jimenez FJ, Vargas C, Gomez P, de Bustos F, Gomez-Escalonilla C, Zurdo M, Tallon A, Martinez-Salio A, Porta-Etessam J, Villanueva C, Arenas J (2002) Cerebrospinal fluid levels of insulin in patients with Alzheimer's disease. Acta Neurol Scand 106, 347-350.

[20] Gil-Bea FJ, Solas M, Solomon A, Mugueta C, Winblad B, Kivipelto M, Ramirez MJ, Cedazo-Minguez A (2010) Insulin levels are decreased in the cerebrospinal fluid of women with prodomal Alzheimer's disease. J Alzheimers Dis 22, 405-413.

[21] Johansson P, Aberg D, Johansson JO, Mattsson N, Hansson $\mathrm{O}$, Ahren B, Isgaard J, Aberg ND, Blennow K, Zetterberg H, Wallin A, Svensson J (2013) Serum but not cerebrospinal fluid levels of insulin-like growth factor-I (IGF-I) and IGF-binding protein-3 (IGFBP-3) are increased in Alzheimer's disease. Psychoneuroendocrinology 38, 1729-1737.

[22] Claxton A, Baker LD, Hanson A, Trittschuh EH, Cholerton B, Morgan A, Callaghan M, Arbuckle M, Behl C, Craft S (2015) Long-acting intranasal insulin detemir improves cognition for adults with mild cognitive impairment or early-stage Alzheimer's disease dementia. J Alzheimers Dis 44, 897-906.

[23] Claxton A, Baker LD, Wilkinson CW, Trittschuh EH, Chapman D, Watson GS, Cholerton B, Plymate SR, Arbuckle M, Craft S (2013) Sex and ApoE genotype differences in treatment response to two doses of intranasal insulin in adults with mild cognitive impairment or Alzheimer's disease. J Alzheimers Dis 35, 789-797.

[24] Benedict C, Kern W, Schultes B, Born J, Hallschmid M (2008) Differential sensitivity of men and women to anorexigenic and memory-improving effects of intranasal insulin. J Clin Endocrinol Metab 93, 1339-1344.

[25] Reger MA, Watson GS, Frey WH 2nd, Baker LD, Cholerton B, Keeling ML, Belongia DA, Fishel MA, Plymate SR, Schellenberg GD, Cherrier MM, Craft S (2006) Effects of intranasal insulin on cognition in memory-impaired older adults: Modulation by APOE genotype. Neurobiol Aging 27, 451-458.

[26] Reger MA, Watson GS, Green PS, Baker LD, Cholerton B, Fishel MA, Plymate SR, Cherrier MM, Schellenberg GD, Frey WH 2nd, Craft S (2008) Intranasal insulin administration dose-dependently modulates verbal memory and plasma amyloid-beta in memory-impaired older adults. J Alzheimers Dis 13, 323-331.

[27] Aalten P, Ramakers IH, Biessels GJ, de Deyn PP, Koek HL, OldeRikkert MG, Oleksik AM, Richard E, Smits LL, van Swieten JC, Teune LK, van der Lugt A, Barkhof F, Teunissen CE, Rozendaal N, Verhey FR, van der Flier WM (2014) The Dutch Parelsnoer Institute-Neurodegenerative diseases; methods, design and baseline results. BMC Neurol 14, 254.

[28] Brand N, Jolles J (1985) Learning and retrieval rate of words presented auditorily and visually. J Gen Psychol 112, 201210.

[29] Rey A (1958) L'examen clinique and psychology, Oxford England: Presses Universitaires.

[30] Van der Elst W, van Boxtel MP, van Breukelen GJ, Jolles J (2005) Rey's verbal learning test: Normative data for 1855 healthy participants aged 24-81 years and the influence of age, sex, education, and mode of presentation. $J$ Int Neuropsychol Soc 11, 290-302. 
[31] Folstein MF, Folstein SE, McHugh PR (1975) "Mini-mental state". A practical method for grading the cognitive state of patients for the clinician. J Psychiatr Res 12, 189-198.

[32] Watson GS, Craft S (2004) Modulation of memory by insulin and glucose: Neuropsychological observations in Alzheimer's disease. Eur J Pharmacol 490, 97-113.

[33] Biessels GJ, Reagan LP (2015) Hippocampal insulin resistance and cognitive dysfunction. Nat Rev Neurosci 16, 660-671.

[34] Rothman KJ (1990) No adjustments are needed for multiple comparisons. Epidemiology 1, 43-46.

[35] Selvin S (1996) Statistical Analysis of Epidemiologic Data, Oxford University Press, New York, NY.

[36] Petrie EC, Cross DJ, Galasko D, Schellenberg GD, Raskind MA, Peskind ER, Minoshima S (2009) Preclinical evidence of Alzheimer changes: Convergent cerebrospinal fluid biomarker and fluorodeoxyglucose positron emission tomography findings. Arch Neurol 66, 632-637.

[37] Ceravolo R, Borghetti D, Kiferle L, Tognoni G, Giorgetti A, Neglia D, Sassi N, Frosini D, Rossi C, Petrozzi L, Siciliano G, Murri L (2008) CSF phosphorylated tau protein levels correlate with cerebral glucose metabolism assessed with PET in Alzheimer's disease. Brain Res Bull 76, 80-84.

[38] Chiaravalloti A, Martorana A, Koch G, Toniolo S, di Biagio D, di Pietro B, Schillaci O (2015) Functional correlates of t-Tau, p-Tau and Abeta(1)(-)(4)(2) amyloid cerebrospinal fluid levels in Alzheimer's disease: A (1)(8)F-FDG PET/CT study. Nucl Med Commun 36, 461-468.

[39] Haense C, Buerger K, Kalbe E, Drzezga A, Teipel SJ, Markiewicz P, Herholz K, Heiss WD, Hampel H (2008) CSF total and phosphorylated tau protein, regional glucose metabolism and dementia severity in Alzheimer's disease. Eur J Neurol 15, 1155-1162.

[40] Vukovich R, Perneczky R, Drzezga A, Forstl H, Kurz A, Riemenschneider M (2009) Brain metabolic correlates of cerebrospinal fluid beta-amyloid 42 and tau in Alzheimer's disease. Dement Geriatr Cogn Disord 27, 474-480.

[41] Okamura N, Arai H, Higuchi M, Tashiro M, Matsui T, Itoh M, Iwatsubo T, Tomita T, Sasaki H (1999) Cerebrospinal fluid levels of amyloid beta-peptide1-42, but not tau have positive correlation with brain glucose metabolism in humans. Neurosci Lett 273, 203-207.

[42] Liguori C, Chiaravalloti A, Sancesario G, Stefani A, Sancesario GM, Mercuri NB, Schillaci O, Pierantozzi M (2016) Cerebrospinal fluid lactate levels and brain [18F]FDG PET hypometabolism within the default mode network in
Alzheimer's disease. Eur J Nucl Med Mol Imaging 43, 2040-2049.

[43] Dumurgier J, Paquet C, Peoc'h K, Lapalus P, MoutonLiger F, Benisty S, Chasseigneaux S, Chabriat H, Hugon J (2011) CSF Abeta1-42 levels and glucose metabolism in Alzheimer's disease. J Alzheimers Dis 27, 845-851.

[44] Blazquez E, Velazquez E, Hurtado-Carneiro V, RuizAlbusac JM (2014) Insulin in the brain: Its pathophysiological implications for States related with central insulin resistance, type 2 diabetes and Alzheimer's disease. Front Endocrinol (Lausanne) 5, 161.

[45] Lauretti E, Li JG, Di Meco A, Pratico D (2017) Glucose deficit triggers tau pathology and synaptic dysfunction in a tauopathy mouse model. Transl Psychiatry 7, e1020.

[46] Dowling NM, Johnson SC, Gleason CE, Jagust WJ, Alzheimer's Disease Neuroimaging Initiative (2015) The mediational effects of FDG hypometabolism on the association between cerebrospinal fluid biomarkers and neurocognitive function. Neuroimage 105, 357-368.

[47] El Khoury NB, Gratuze M, Papon MA, Bretteville A, Planel E (2014) Insulin dysfunction and Tau pathology. Front Cell Neurosci 8, 22.

[48] Clegg DJ, Riedy CA, Smith KA, Benoit SC, Woods SC (2003) Differential sensitivity to central leptin and insulin in male and female rats. Diabetes 52, 682-687.

[49] Hallschmid M, Benedict C, Schultes B, Fehm HL, Born J, Kern W (2004) Intranasal insulin reduces body fat in men but not in women. Diabetes 53, 3024-3029.

[50] Asthana S (2003) Estrogen and cognition: The story so far. J Gerontol A Biol Sci Med Sci 58, 322-323.

[51] Krug R, Benedict C, Born J, Hallschmid M (2010) Comparable sensitivity of postmenopausal and young women to the effects of intranasal insulin on food intake and working memory. J Clin Endocrinol Metab 95, E468-472.

[52] Reger MA, Watson GS, Green PS, Wilkinson CW, Baker LD, Cholerton B, Fishel MA, Plymate SR, Breitner JC, DeGroodt W, Mehta P, Craft S (2008) Intranasal insulin improves cognition and modulates beta-amyloid in early AD. Neurology 70, 440-448.

[53] Boden G, Ruiz J, Urbain JL, Chen X (1996) Evidence for a circadian rhythm of insulin secretion. Am J Physiol 271, E246-252.

[54] Cedernaes J, Osorio RS, Varga AW, Kam K, Schioth HB, Benedict C (2017) Candidate mechanisms underlying the association between sleep-wake disruptions and Alzheimer's disease. Sleep Med Rev 31, 102-111. 